\title{
Fruit and vegetable consumption among migrants in Switzerland
}

\author{
Thomas Volken ${ }^{1, *}$, Peter Rüesch ${ }^{1}$ and Jürg Guggisberg ${ }^{2}$ \\ 'School of Health Professions, Zurich University of Applied Sciences, Technikumstrasse 71, POB 8401, \\ Winterthur, Switzerland: ${ }^{2}$ Büro für Arbeits- und Sozialpolitische Studien BASS, Bern, Switzerland
}

Submitted 23 September 2011: Final revision received 20 March 2012: Accepted 30 March 2012: First published online 14 May 2012

\begin{abstract}
Objective: To assess the relative risk of low daily fruit and vegetable consumption for six large migrant groups in Switzerland.

Design: Cross-sectional health survey carried out 2007 (Swiss Health Survey) and 2010 (Swiss Migrant Health Survey) in Switzerland. Multinomial logistic regression models were used to estimate relative risk rates (RRR) of migrants relative to Swiss nationals.

Setting: Data obtained from representative samples of Swiss and foreign nationals living in Switzerland.

Subjects: A random sample ( $n$ 14637) of the Portuguese, German, Italian, Turkish, Serbian, Kosovan and Swiss permanent resident adult population (17-64 years old) was interviewed.

Results: The proportion of participants who adhered to the recommended fruit and vegetable consumption was below one-third in all study populations. Compared with Swiss nationals, the relative risk of low daily fruit and vegetable intake relative to recommended intake was higher in Turkish nationals $(\mathrm{RRR}=2 \cdot 92$, $95 \%$ CI 1.91, 4.48; $P=0 \cdot 0000$ ) and Kosovan nationals (RRR $=4 \cdot 76,95 \%$ CI $3 \cdot 01$, 7.55; $P=0 \cdot 0000)$. The respective relative risks of Portuguese, Serbian, German and Italian nationals were not significantly different from the Swiss reference group. Conclusions: Initiatives for the promotion of fruit and vegetable consumption should continue to address the population at large. At the same time, programmes that are tailored to the specific needs of migrants from Turkey and Kosovo should be considered.
\end{abstract}

Non-communicable diseases, especially CVD, cancer, obesity and type 2 diabetes mellitus, currently kill more people every year than any other cause of death. Fruit and vegetables are an important component of a healthy diet and, if consumed daily in sufficient amounts, could help prevent major diseases such as CVD and certain cancers ${ }^{(1)}$.

Several studies have found associations between a higher intake of fruit and vegetables and a reduced risk of CVD mortality ${ }^{(2-8)}$. Similarly, a higher daily intake of fruit and vegetables is associated with a reduced risk of some types of cancer $^{(9-14)}$.

In 2001, the Swiss Cancer League, supported by the Swiss Federal Office of Public Health and Health Promotion Switzerland started an ongoing prevention campaign 'Five servings of fruit and vegetables a day' in order to promote an adequate daily consumption of fruit and vegetables among the population of Switzerland. In 2007, $29 \cdot 3 \%$ of the Swiss population aged 15 years and older reported to consume the recommended five portions daily ${ }^{(15)}$. Comparably little is known about the factors that are associated with fruit and vegetable consumption in
Switzerland. There is some evidence that women and older age cohorts have a higher propensity to consume fruit and vegetables compared with men and younger age cohorts $^{(15,16)}$. To our knowledge, no population-based study so far has assessed the fruit and vegetable consumption of adult migrants in Switzerland, despite the fact that migrants accounted for 1.7 million people or $22 \%$ of the permanent resident population of Switzerland in $2009^{(17)}$. Migrants are an important group and prevention campaigns may need to be tailored to specific migrant groups and their needs. A large body of literature on migration and health suggests that cultural patterns shape physical activity, body images, dietary intake and food preferences ${ }^{(18-24)}$. However, the evidence on dietary patterns is mixed ${ }^{(25)}$. While some studies report that migrant status is associated with a low daily intake of fruit and vegetables or adverse dietary patterns ${ }^{(26,27)}$, others report that migrant status is associated with a higher daily intake or beneficial dietary patterns compared with the native population of the host country ${ }^{(28-32)}$. Given that the mixed evidence partially reflects culture-specific dietary practices as well as acculturation to the dietary 
practices of the host countries, we may expect different patterns of fruit and vegetable consumption among migrants in Switzerland as well. The aim of the present study was to assess the risk of low daily intake of fruit and vegetables among six large migrant groups in Switzerland and thereby serve as a guide for effective fruit and vegetable promotion interventions.

\section{Methods}

\section{Study design}

The study is a population-based cross-sectional health survey carried out in 2007 and 2010 in Switzerland. Ethical approval for the study was not required.

\section{Study population and data}

Data from the 2010 Swiss Migrant Health Survey (SMHS) and the 2007 Swiss Health Survey (SHS) were obtained from the Swiss Federal Office of Public Health (www.bag. admin.ch). The SMHS is a cross-sectional, populationbased telephone survey which intends to monitor health trends in a representative sample of migrants and has been carried out in 2004 and 2010 so far. Due to economic constraints, the SMHS is limited to a selected number of nationalities. The core study population in 2010 comprised permanent residents from Portugal, Turkey, Serbia and Kosovo ( $n$ 1800). Participants aged $17-74$ years were chosen by stratified random sampling (gender, country of birth, duration of stay) from the database of the central immigration information system. The computer-aided telephone interviews were carried out in Portuguese, Turkish, Serbian, Albanian, German and French. Data on Swiss, German and Italian nationals from the 2007 SHS were pooled with the 2010 SMHS data. The SHS was first conducted in 1992 and is repeated every five years. The 2007 sample included over 19000 participants aged 15 years or older. Participants were randomly selected within private households and computer-aided telephone interviews were conducted in German, French and Italian. People who did not speak any of these languages or people who had insufficient language skills were excluded from the SHS.

The pooled sample represents $58 \cdot 2 \%$ of the migrant population as well as nationals from Switzerland. Of the 1.7 million migrants in Switzerland, $17 \cdot 0 \%$ are Italian, $14.9 \%$ are German, $12.0 \%$ are Portuguese, $5.4 \%$ are French, $3 \cdot 6 \%$ are Spanish, $6 \cdot 9 \%$ are Serbians, $4 \cdot 1 \%$ are Turkish and $3 \cdot 3 \%$ are Kosovan ${ }^{(33)}$. Migrants from France and Spain were not included because of their small sample size in the SHS. Since all interviews of participants in the pooled sample were conducted in their respective native language, selection bias due to insufficient language skills has been mitigated; i.e. the selection process is less likely to favour migrants who are well-integrated, well-educated and have been living in Switzerland for a long period of time.
For the purposes of the present study, the initial pooled sample was further narrowed down to include only those aged 17-64 years because only a few migrants in the SMHS sample were over 64 years old. The total sample size amounts to 14637 . The sample size by nationality and further characteristics of the pooled SMHS and SHS sample is shown in Table 1.

\section{Target outcome, predictor and covariates}

Daily fruit and vegetable intake (FVI), the target outcome of the study, was derived from self-reported data. Participants were asked to estimate their average daily consumption of fruit by answering the question: 'How many servings of fruit and fruit juice do you have on average per day? One serving corresponds to the size of your fist (approximately $120 \mathrm{~g}$ ). In the case of fruit juice, one serving corresponds to a medium size glass'. Similarly, a question relating to the average consumption of vegetables was asked: 'How many servings of vegetable and vegetable juice (without potatoes or sweet corn) do you have on average per day? One serving corresponds to the size of your fist (approximately $120 \mathrm{~g}$ ). In the case of vegetable juice, one serving corresponds to a medium size glass'. The choice of answers included: (i) 'none'; (ii) 'less than 1 serving per day'; (iii) '1-2 servings per day'; (iv) '3-4 servings per day'; and (v) '5 or more servings per day'. FVI was calculated by adding up the self-reported frequency of fruit and vegetable consumption* $^{*}{ }^{(15)}$. Participants were then assigned to the following three categories: (i) recommended FVI (FVI $\geq 5$ servings of fruit and vegetables/d) $\uparrow$; (ii) medium FVI $(2 \leq \mathrm{FVI} \leq 4)$; and (iii) low FVI $(0 \leq \mathrm{FVI}<2)$. Although these questions have been used before ${ }^{(15,16,34)}$, the construct and content validity of this instrument have not been formally assessed so far. We conducted a linguistic validation process that included a conceptual analysis of all original instruments in collaboration with the translators, as well as forward and backward translations and a review of the backward translation.

For the primary predictor, the country of origin, the following were considered: Portugal, Turkey, Serbia, Kosovo, Germany, Italy and Switzerland. Double citizens who acquired Swiss nationality through naturalization were considered Swiss. Covariates included: age, gender, socio-economic status (SES) and dwelling zone (rural/ urban). SES comprised three indicators: education (basic, secondary and university), employment (economically active, no/yes) and housing situation (rooms per person).

\footnotetext{
* For the summation process the following values were assigned to the categories: $0=$ 'none'; $0 \cdot 5=$ 'less than 1 serving per day'; $1 \cdot 5={ }^{\prime} 1-2$ servings per day'; $3 \cdot 5=3-4$ servings per day'; and $5=$ ' 5 or more servings per day'.

$\uparrow$ The category 'recommended FVI' reflects the amount of daily fruit and vegetable intake which is currently promoted by the Swiss Federal Office of Public Health (http://www.bag.admin.ch/themen/ernaehrung_bewegung) and the Swiss Cancer League.
} 


\section{Missing data}

Missing data were generally rare on all variables. For the predictor and two covariates, no data were missing and for the remaining covariates $0.5 \%$ or less of the data were missing within each nationality. Missing data on FVI, the dependent variable, amounted to $2 \cdot 6 \%$ and there were no substantial differences in the amount of missing data between nationalities. Overall, 3\% of the data were missing. Our analysis of missing-value patterns also revealed no systematic relationships between FVI and the predictor or the covariates. We therefore assumed that the data were missing completely at random and applied listwise deletion which gives valid inferences under this assumption $^{(35)}$.

\section{Weighting}

The original expansion weights of the SMHS and SHS were used for all population estimates. The weights take into account the different sampling strategies which have been used to sample migrants and Swiss nationals and they allow correct estimates of population parameters for migrants and Swiss nationals.

\section{Statistical analysis}

We used the STATA statistical software package version $11 \cdot 2$ for all statistical analyses. We report relative risk ratios (RRR) for different nationalities from multinomial logistic regression models (mlogit) adjusting for age, gender, SES and dwelling zone. We also report corresponding 95\% confidence intervals and $P$ values. To incorporate information on the appropriate weights and sampling units for correct variance estimation, all statistical analyses were carried out using STATA's command for complex surveys (svy prefix). Statistical significance was established at $P \leq 0 \cdot 05$.

\section{Results}

We found different prevalence rates of low, medium and recommended FVI between migrants and nationals of Switzerland (Table 2): 30·3\% (95\% CI 29.2, 31·3) of Swiss nationals adhered to the recommended FVI. The respective prevalence was significantly lower in nationals from Turkey $(22.9 \%$; $95 \%$ CI 18.2, 28.2; $P=0 \cdot 0044)$, Serbia (22.4\%; $95 \%$ CI 17·4, 28.2; $P=0 \cdot 0046)$, Kosovo $(18 \cdot 3 \%$; 95\% CI $13 \cdot 7,24 \cdot 0 ; P=0 \cdot 0000)$ and Italy $(23 \cdot 5 \% ; 95 \%$ CI $18.5,29.4 ; P=0.0166)$. In contrast, the prevalence of recommended FVI of German (30.4\%; $95 \%$ CI 24.5, 37.1; $P=0.9661)$ and Portuguese (28.2\%; 95\% CI 23.7, 33.2; $P=0 \cdot 4029)$ nationals did not differ significantly from that of Swiss nationals.

The proportion of Swiss nationals with medium FVI amounted to $62 \cdot 6 \%(95 \% \mathrm{CI} 61 \cdot 5,63 \cdot 7)$. The respective proportion was found to be lower in Turkish nationals (52.9\%; 95\% CI 47.0, 58.8; $P=0 \cdot 0015)$ and nationals 
Table 2 Population estimates of fruit and vegetable consumption of participants aged 17-64 years by nationality (2010 Swiss Migrant Health Survey and 2007 Swiss Health Survey 2007)*

\begin{tabular}{|c|c|c|c|c|c|c|c|c|c|}
\hline & \multicolumn{3}{|c|}{$0-2$ servings/dt } & \multicolumn{3}{|c|}{$2-4$ servings/dt } & \multicolumn{3}{|c|}{$\geq 5$ servings $/ \mathrm{d} \dagger$} \\
\hline & $n \ddagger$ & $\%$ & $95 \% \mathrm{Cl}$ & $n \ddagger$ & $\%$ & $95 \% \mathrm{Cl} \ddagger$ & $n \ddagger$ & $\%$ & $95 \% \mathrm{Cl}$ \\
\hline Portugal & 14247 & $9 \cdot 1$ & $6 \cdot 4,12 \cdot 9$ & 97853 & $62 \cdot 7$ & $57 \cdot 4,67 \cdot 7$ & 44007 & $28 \cdot 2$ & $23 \cdot 7,33 \cdot 2$ \\
\hline Turkey & 12698 & $24 \cdot 2$ & $19 \cdot 6,29 \cdot 5$ & 27727 & $52 \cdot 9$ & $47 \cdot 0,58 \cdot 8$ & 11956 & $22 \cdot 9$ & $18 \cdot 2,28 \cdot 2$ \\
\hline Serbia & 4060 & $10 \cdot \overline{5}$ & $7 \cdot 1,15 \cdot 3$ & 25950 & $67 \cdot 1$ & $60 \cdot 6,73 \cdot 1$ & 8639 & $22 \cdot 4$ & $17 \cdot 4,28 \cdot 2$ \\
\hline Kosovo & 27328 & $32 \cdot 3$ & $26 \cdot 5,38 \cdot 8$ & 41750 & $49 \cdot 4$ & $42 \cdot 8,56 \cdot 0$ & 15459 & $18 \cdot 3$ & $13 \cdot 7,24 \cdot 0$ \\
\hline Germany & 9593 & 6.0 & $3 \cdot 8,9 \cdot 3$ & 101542 & $63 \cdot 6$ & $56 \cdot 9,69 \cdot 8$ & 48575 & $30 \cdot 4$ & $24 \cdot 5,37 \cdot 1$ \\
\hline Italy & 16668 & $10 \cdot 0$ & $6 \cdot 9,14 \cdot 4$ & 110581 & $66 \cdot 5$ & $60 \cdot 1,72 \cdot 3$ & 39055 & 23.5 & $18 \cdot 5,29 \cdot 4$ \\
\hline Switzerland & 256372 & $7 \cdot 1$ & $6 \cdot 5,7 \cdot 7$ & 2262312 & $62 \cdot 6$ & $61 \cdot 5,63 \cdot 7$ & 1093676 & $30 \cdot 3$ & $29 \cdot 2,31 \cdot 3$ \\
\hline
\end{tabular}

*Data were weighted using the weights of the Swiss Migrant Health Survey/Swiss Health Survey.

tOne serving corresponds to approximately $120 \mathrm{~g}$. In the case of juice, one serving corresponds to a medium size glass, approximately $150 \mathrm{ml}$. ‡Estimated number of participants in the population.

from Kosovo (49.4\%; $95 \%$ CI $42 \cdot 8,56 \cdot 0 ; P=0 \cdot 0001)$, whereas the proportion of Swiss nationals with medium FVI was not different from nationals from Portugal (62.7\%; 95\% CI 57.4, 67.7; $P=0 \cdot 9832)$, Serbia (67.1\%; 95\% CI 60.6, 73.1; $P=0 \cdot 1637)$, Germany $(63 \cdot 6 \% ; 95 \%$ CI $56 \cdot 9,69 \cdot 8 ; P=0 \cdot 7768)$ and Italy $(66 \cdot 5 \% ; 95 \%$ CI $60 \cdot 1$, 72.3; $P=0 \cdot 2236$ ).

Low FVI was more common in nationals from Turkey (24.2\%; 95\% CI 19.6, 29.5; $P=0.0000)$ and Kosovo (32.3\%; 95\% CI 26.5, 38.8; $P=0.0000)$ than in Swiss nationals $(7 \cdot 1 \% ; 95 \% \mathrm{CI} 6 \cdot 5,7 \cdot 7)$. In contrast, the proportion of Swiss nationals with low FVI did not differ from Portuguese $(9 \cdot 1 \% ; 95 \%$ CI $6 \cdot 4,12 \cdot 9 ; P=0 \cdot 2211)$, Serbian $(10 \cdot 5 \% ; 95 \%$ CI $7 \cdot 1,15 \cdot 3 ; P=0 \cdot 1052)$, German (6.0\%; $95 \%$ CI $3 \cdot 8,9 \cdot 3 ; P=0 \cdot 4339)$ and Italian $(10 \cdot 0 \%$; $95 \%$ CI $6 \cdot 9,14 \cdot 4)$ nationals.

Multivariate analysis adjusting for age, gender, SES and dwelling zone generally confirmed these initial results (Table 3). Compared with Swiss nationals, the relative risk of low FVI $v$. recommended FVI was higher in Turkish nationals $(\mathrm{RRR}=2 \cdot 92,95 \% \mathrm{CI} 1.91,4 \cdot 48 ; \quad P=0 \cdot 0000)$ and Kosovo nationals (RRR $=4 \cdot 76,95 \%$ CI 3.01, 7.55; $P=0 \cdot 0000)$. The respective relative risks of Portuguese (RRR $=0 \cdot 70,95 \%$ CI $0 \cdot 42,1 \cdot 17 ; \quad P=0 \cdot 1750)$, Serbian (RRR $=1 \cdot 42,95 \%$ CI $0 \cdot 82,2 \cdot 46 ; P=0 \cdot 2140)$, German (RRR $=0 \cdot 87,95 \%$ CI $0.51,1 \cdot 50 ; P=0 \cdot 6180)$ and Italian $(\mathrm{RRR}=1 \cdot 32,95 \% \mathrm{CI} 0 \cdot 80,2 \cdot 18 ; P=0 \cdot 2740)$ nationals were not significantly different from the Swiss reference group.

Migrants from different nationalities and Swiss nationals showed similar relative risks of medium FVI $v$. recommended FVI; none of the nation-specific RRR attained statistical significance.

The relative risk of low FVI decreased with age $(\mathrm{RRR}=0 \cdot 98,95 \% \mathrm{CI} 0 \cdot 98,0 \cdot 99 ; P=0 \cdot 0000)$. That is, older participants were more likely to consume the recommended five portions daily. Similarly, women were more likely to eat greater amounts of fruits and vegetables than men. Correspondingly, women exhibited a lower risk for low or medium FVI $v$. recommended FVI $(\mathrm{RRR}=0 \cdot 22$, $95 \%$ CI $0 \cdot 18,0 \cdot 26 ; P=0 \cdot 0000$ and $\mathrm{RRR}=0 \cdot 41,95 \% \mathrm{CI}$ $0 \cdot 37,0 \cdot 45 ; P=0 \cdot 0000$, respectively). Also, participants with secondary education or a university degree were more likely to consume greater amounts of fruits and vegetables than those with a basic education (secondary education: $\mathrm{RRR}=0 \cdot 47,95 \% \mathrm{CI} 0 \cdot 35,0 \cdot 62 ; P=0 \cdot 0000$ and $\mathrm{RRR}=0 \cdot 75,95 \%$ CI $0 \cdot 62,0 \cdot 90 ; P=0 \cdot 0020$ for low and medium FVI, respectively, $v$. recommended FVI; university degree: $\mathrm{RRR}=0 \cdot 27,95 \% \mathrm{CI} 0 \cdot 19,0 \cdot 37 ; P=0 \cdot 0000$ and RRR $=0.56,95 \%$ CI $0.46,0.69 ; P=0.0000$ for low and medium FVI, respectively, $v$. recommended FVI). Economically active participants were slightly more likely to have a higher FVI than those who were not economically active (medium FVI: RRR $=1 \cdot 19$, $95 \%$ CI 1·05, 1·35; $P=0 \cdot 0050$; low FVI: NS). Finally, neither housing situation nor the specific dwelling zone was significantly associated with low or medium FVI $v$, recommended FVI.

Several studies show that migrants often adopt the dietary practices of the host country ${ }^{(30,36-38)}$. Consequently, fruit and vegetable consumption of migrants in Switzerland may also be influenced by dietary acculturation. In order to control for potential acculturation effects, length of time in Switzerland (years) was added as a covariate to the model previously discussed and the model was re-estimated using migrant data only. However, length of time in Switzerland had no statistically significant association with FVI (results not shown).

\section{Discussion}

So far, population-based studies on the fruit and vegetable consumption among migrants in Switzerland are rare and have been based mainly on data of the $\mathrm{SHS}^{(39)}$. However, the SHS data have limitations with regard to the study of migrants. First, interviews are carried out only in German, French and Italian. Second, the sampling process does not include any migrant-specific characteristics, e.g. the country of origin. Consequently, the SHS data potentially favour well-integrated, well-educated and well-assimilated migrants and potentially omit vulnerable groups of migrants. Furthermore, the sample size for specific groups of migrants, e.g. Turkish migrants, tends to be very small. 
Table 3 Multinomial regression analysis of factors associated with daily fruit and vegetable consumption, participants aged 17-64 years (2010 Swiss Migrant Health Survey and 2007 Swiss Health Survey) ${ }^{\star}$

\begin{tabular}{|c|c|c|c|}
\hline n 14637; base outcome $\geq 5$ servings/dt & $\mathrm{RRR}$ & $P$ value & $95 \% \mathrm{Cl}$ \\
\hline \multicolumn{4}{|l|}{$0-2$ servings/d (low FVI)† } \\
\hline \multicolumn{4}{|l|}{ Nationality (ref. $=$ Switzerland) } \\
\hline Portugal & $0 \cdot 70$ & $0 \cdot 1750$ & $0 \cdot 42,1 \cdot 17$ \\
\hline Turkey & $2 \cdot 92$ & 0.0000 & $1 \cdot 91,4 \cdot 48$ \\
\hline Serbia & $1 \cdot 42$ & 0.2140 & $0.82,2 \cdot 46$ \\
\hline Kosovo & $4 \cdot 76$ & 0.0000 & $3 \cdot 01,7 \cdot 55$ \\
\hline Germany & $0 \cdot 87$ & $0 \cdot 6180$ & $0.51,1.50$ \\
\hline Italy & $1 \cdot 32$ & 0.2740 & $0 \cdot 80,2 \cdot 18$ \\
\hline Age (years) & 0.98 & 0.0000 & $0.98,0.99$ \\
\hline Female (ref. $=$ male) & 0.22 & 0.0000 & $0.18,0.26$ \\
\hline \multicolumn{4}{|l|}{ Education (ref. = basic) } \\
\hline Secondary & 0.47 & 0.0000 & $0.35,0.62$ \\
\hline University & 0.27 & 0.0000 & $0.19,0.37$ \\
\hline Economically active (ref. = no) & 1.09 & 0.4200 & $0 \cdot 88,1 \cdot 36$ \\
\hline Housing situation (rooms/person) & $1 \cdot 09$ & $0 \cdot 0680$ & $0 \cdot 99,1 \cdot 20$ \\
\hline Rural dwellerł (ref. $=$ urban dweller) & 0.91 & $0 \cdot 3450$ & $0 \cdot 75,1 \cdot 10$ \\
\hline \multicolumn{4}{|l|}{ 2-4 servings/d (medium FVI)† } \\
\hline \multicolumn{4}{|l|}{ Nationality (ref. = Switzerland) } \\
\hline Portugal & $0 \cdot 80$ & $0 \cdot 1090$ & $0 \cdot 61,1 \cdot 05$ \\
\hline Turkey & 0.96 & 0.7970 & $0.69,1.33$ \\
\hline Serbia & $1 \cdot 28$ & $0 \cdot 1670$ & $0.90,1 \cdot 82$ \\
\hline Kosovo & $1 \cdot 10$ & 0.6390 & $0 \cdot 74,1 \cdot 64$ \\
\hline Germany & $1 \cdot 02$ & $0 \cdot 8910$ & $0.75,1 \cdot 40$ \\
\hline Italy & $1 \cdot 15$ & $0 \cdot 3980$ & $0.83,1.60$ \\
\hline Age (years) & $1 \cdot 00$ & $0 \cdot 4830$ & $0.99,1 \cdot 00$ \\
\hline Female $($ ref. $=$ male $)$ & $0 \cdot 41$ & 0.0000 & $0.37,0.45$ \\
\hline \multicolumn{4}{|l|}{ Education (ref. = basic) } \\
\hline Secondary & $0 \cdot 75$ & 0.0020 & $0.62,0.90$ \\
\hline University & 0.56 & $0 \cdot 0000$ & $0.46,0.69$ \\
\hline Economically active (ref. $=$ no) & $1 \cdot 19$ & 0.0050 & $1 \cdot 05,1 \cdot 35$ \\
\hline Housing situation (rooms/person) & 0.99 & 0.6150 & $0.94,1.04$ \\
\hline Rural dweller $\ddagger$ (ref. $=$ urban dweller) & 0.97 & 0.5410 & $0 \cdot 87,1 \cdot 08$ \\
\hline
\end{tabular}

RRR, relative risk ratio; ref., referent category.

*Data were weighted using the weights of the Swiss Migrant Health Survey/Swiss Health Survey.

tOne serving corresponds to approximately $120 \mathrm{~g}$. In the case of juice, one serving corresponds to a medium size glass, approximately $150 \mathrm{ml}$. $\neq$ Classification according to the Swiss Federal Statistical Office ${ }^{(61)}$. Urban areas include isolated cities ( $\geq 10000$ inhabitants) and urban agglomerations ( $\geq 20000$ inhabitants).

To our knowledge, the present study is the first which uses the 2010 SMHS data to assess fruit and vegetable consumption among migrants and hence to use a survey that has been specially designed to collect public-healthrelated data on migrants in Switzerland. Since all SMHS interviews were conducted in the participants' respective native languages, selection bias due to insufficient language skills has been mitigated.

Overall, the proportion of participants who ate five or more servings of fruit and vegetables daily was below one-third in all study groups. The highest rate of recommended FVI was little more than 30\% (German and Swiss nationals); the lowest rate amounted to little more than $18 \%$ (Kosovo nationals). Hence, fruit and vegetable promotion initiatives should continue to address the population of Switzerland at large in order to increase fruit and vegetable consumption. Our results further suggest that some migrant groups are at particular risk of low daily fruit and vegetable consumption. For migrants from Turkey and Kosovo relative to Swiss nationals, the relative risk of low FVI $v$. recommended FVI was substantially higher. In contrast, the respective relative risk for German, Portuguese, Serbian and Italian nationals did not significantly differ from Swiss nationals. In sum, substantial differences in the relative risk for low FVI $v$. recommended FVI existed between Swiss nationals and migrants from Turkey and Kosovo, and these differences cannot be attributed to age, gender, SES or dwelling zone since we adjusted for these factors.

Previous studies reported that women and older age cohorts consume fruit and vegetables more often than men and younger age cohorts; and that participants with a higher level of education tend to consume more fruit and vegetables than those with a low level of education $^{(15,16,25,40,41)}$. Generally, our findings correspond to the results of other studies. However, an increase in age by 1 year only reduced the relative risk for low FVI $v$. recommended FVI but did not significantly reduce the relative risk for low FVI $v$. medium FVI in our study. Secondary and university education relative to basic education substantially reduced the risk of both low FVI and medium FVI $v$. recommended FVI. With regard to sociodemographic factors and SES, these findings suggest that health promotion programmes should specifically address younger age cohorts, the male population and people with low education level. 


\section{Limitations of the study}

The present study has several limitations inherent to all FFQ. First, reporting the amount of food consumption is a difficult cognitive task ${ }^{(42)}$. Although the SMHS and SHS questions on FVI included guidelines on size by specifying the weight of one serving (120 g of fruit/vegetables or one medium glass of fruit/vegetable juice), it remains unclear to what extent participants were able to adequately assess the size and weight of food consumed. Second, recalling the average servings of fruit and vegetables consumed each day is also a difficult task. Memory can be quite unreliable or may be influenced by more recent intakes of fruit and vegetables. Third, social desirability may be an issue. Participants may over-report their daily fruit and vegetable consumption because high intake is promoted as a healthy habit. Finally, questions on fruit and vegetables could be culture-sensitive ${ }^{(43)}$, i.e. the concepts of fruit and vegetables may be different or there may be systematic covariations of cultural background and the potential sources of bias mentioned above. More specifically, fruit and vegetables in mixed dishes could not have been considered by some individuals. The European Prospective Investigation into Cancer and Nutrition (EPIC) showed that the intake of vegetables from mixed vegetable dishes varies substantially by country $(12-54 \%)^{(42,44)}$ and a review of brief fruit and vegetable surveys concluded that inclusion of the consumption of mixed vegetable dishes may enhance the validity of the intruments ${ }^{(45)}$. Several validation studies came to the conclusion that FFQ tend to overestimate the mean intake as compared with repeated $24 \mathrm{~h}$ dietary recalls, which served as the reference method for assessing intake ${ }^{(46,47)}$. These potential biases suggest that the present results should be interpreted with caution. However, although FFQ may be affected by scaling biases and random error when estimating absolute FVI, they seem to be valid as means of ranking participants by level of FVI. Finally, it is also plausible that mixed dishes, where the major components are fruit or vegetables, are considered when participants report their consumption in $\mathrm{FFQ}^{(42)}$.

\section{Potential interventions}

Eating behaviour is highly complex and has been described as resulting from the interplay of individual motivation, ability and environmental factors ${ }^{(48)}$. So far, the reasons why Turks and Kosovans have a higher risk of low FVI are unclear and more research is needed. However, we assume that limited access to healthy food in general is not an issue in Switzerland (physical environment). A dense network of supermarkets and local grocers offers fresh fruit and vegetables at reasonable prices. It is also well known that energy-dense diets are associated with lower diet costs ${ }^{(49,50)}$. But fruit and vegetables are generally affordable for those with small household budgets ${ }^{(15)}$ (economic environment) and a major supermarket chain, which supports the Five a Day campaign of the Swiss Federal Office of Health, regularly makes special offers on fruit and vegetables. Moreover, it seems unlikely that migrants from Turkey and the Kosovo are less aware of FVI than other migrant groups or native Swiss because information and materials are available in foreign languages. However, diet-related public health initiatives which address migrants specifically do not exist to date. Since neither awareness nor the physical or economic environment appears to be a barrier specific to migrants from Turkey and Kosovo, we propose that nutrition education initiatives embedded in migrant cultural clubs may be a promising approach to increase fruit and vegetable consumption. These clubs could serve as a basis for peer health educators who offer cookery courses, food tasting and other activities which revive the healthy cooking traditions of Turkey and Kosovo. Food tasting and cookery courses, in conjunction with the proposed social setting, target different but complementing determinants of FVI which are now discussed briefly.

First, there is evidence that food preferences can be learned $^{(51-55)}$. The opportunity to repeatedly taste traditional and novel fruit and vegetable dishes can increase the liking for these dishes. Hence food tastings can help to change food preferences and can increase the motivation to consume more fruit and vegetables. Second, several studies that systematically review the determinants of FVI show that self-efficacy, i.e. people's belief in their ability to succeed in certain situations ${ }^{(56)}$, and nutritional knowledge are positively associated with $\mathrm{FVI}^{(48,57-59)}$. Cookery courses can increase practical knowledge on how to prepare novel fruit and vegetable dishes and healthier variations of traditional dishes (healthy alternatives). Similarly, they can increase knowledge on recommended intake levels and awareness of personal intake. Cookery courses help to enable voluntary dietary change by enhancing people's ability, i.e. skills and capabilities. Third, food tasting and cookery courses take place in migrant cultural clubs. The latter can be considered a micro-environmental setting of the sociocultural environment, which refers to the migrant community's attitudes, beliefs and values related to food and physical activity ${ }^{(60)}$. Food-related activities within these micro-environmental settings may not only change people's motivation and ability, but may also help to develop and foster new dietary norms and cooking traditions when people start to incorporate the new dietary practices into their everyday life. Moreover, peer social networks may help to further diffuse new dietary practices into the broader sociocultural environment when families, friends and neighbours are regularly confronted with healthy fruit and vegetable dishes.

\section{Conclusions}

In summary, the proportion of participants who adhered to the recommended fruit and vegetable consumption was below one-third in all study populations. Migrants 
from Turkey and Kosovo relative to Swiss nationals are at particular risk of low daily fruit and vegetable consumption relative to the recommended daily intake. Initiatives for the promotion of fruit and vegetable consumption should continue to address the population at large. At the same time, programmes that are tailored to the specific needs of migrants from Turkey and Kosovo should be considered.

\section{Acknowledgements}

This study was supported by the Swiss Federal Office of Public Health and the Swiss Federal Office for Migration, grant number $09.005923 / 704.0001 /-330$. The authors declare that they have no conflict of interest. T.V. contributed most of the writing and the analysis. P.R. and J.G. contributed to the writing and editorial review.

\section{References}

1. World Health Organization/Food and Agriculture Organization of the United Nations (2005) Fruit and Vegetables for Health. Report of a Joint FAO/WHO Workshop, 1-3 September 2004, Kobe, Japan. Geneva: WHO and FAO.

2. Crowe FL, Roddam AW, Key TJ et al. (2011) Fruit and vegetable intake and mortality from ischaemic heart disease: results from the European Prospective Investigation into Cancer and Nutrition (EPIC)-Heart study. Eur Heart J 32, 1235-1243.

3. Dauchet L, Montaye M, Ruidavets JB et al. (2010) Association between the frequency of fruit and vegetable consumption and cardiovascular disease in male smokers and non-smokers. Eur J Clin Nutr 64, 578-586.

4. Dauchet L, Amouyel P \& Dallongeville J (2009) Fruits, vegetables and coronary heart disease. Nat Rev Cardiol 6, 599-608.

5. Oude Griep LM, Geleijnse JM, Kromhout D et al. (2010) Raw and processed fruit and vegetable consumption and 10 -year coronary heart disease incidence in a populationbased cohort study in the Netherlands. PLoS One 5, e13609.

6. Scarborough P, Nnoaham KE, Clarke D et al. (2012) Modelling the impact of a healthy diet on cardiovascular disease and cancer mortality. I Epidemiol Community Health 66, 420-426.

7. Tyrovolas S \& Panagiotakos DB (2010) The role of Mediterranean type of diet on the development of cancer and cardiovascular disease, in the elderly: a systematic review. Maturitas 65, 122-130.

8. Zhang X, Shu XO, Xiang YB et al. (2011) Cruciferous vegetable consumption is associated with a reduced risk of total and cardiovascular disease mortality. Am J Clin Nutr 94, 240-246.

9. Zhang CX, Ho SC, Fu JH et al. (2011) Dietary patterns and breast cancer risk among Chinese women. Cancer Causes Control 22, 115-124.

10. Steevens J, Schouten LJ, Goldbohm RA et al. (2011) Vegetables and fruits consumption and risk of esophageal and gastric cancer subtypes in the Netherlands Cohort Study. Int J Cancer 129, 2681-2683.

11. Richman EL, Carroll PR \& Chan JM (2012) Vegetable and fruit intake after diagnosis and risk of prostate cancer progression. Int J Cancer 131, 201-210.

12. Ott JJ, Ullrich A, Mascarenhas M et al. (2011) Global cancer incidence and mortality caused by behavior and infection. J Public Health (Oxf) 33, 223-233.
13. Aune D, Lau R, Chan DS et al. (2011) Nonlinear reduction in risk for colorectal cancer by fruit and vegetable intake based on meta-analysis of prospective studies. Gastroenterology 141, 106-118.

14. Bravi F, Polesel J, Bosetti C et al. (2011) Dietary intake of selected micronutrients and the risk of pancreatic cancer: an Italian case-control study. Ann Oncol 22, 202-206.

15. Lamprecht M \& Stamm H (2009) Monitoring der langfristigen Strategie von Gesundheitsförderung Schweiz. Indikatoren zum Bereich „Gesundes Körpergewicht". Bern: Health Promotion Switzerland.

16. Eichholzer M, Bernasconi F, Jordan $\mathrm{P}$ et al. (2005) Ernährung in der Schweiz. Resultate der Schweizerischen Gesundheitsbefragung 2002. Praxis 94, 1713-1721.

17. Bundesamt für Statistik (2010) Die Bevölkerung der Schweiz 2009. Neuchâtel: Swiss Federal Statistical Office.

18. Caperchione CM, Kolt GS \& Mummery WK (2009) Physical activity in culturally and linguistically diverse migrant groups to Western society: a review of barriers, enablers and experiences. Sports Med 39, 167-177.

19. Ceballos N \& Czyzewska M (2010) Body image in Hispanic/Latino vs. European American adolescents: implications for treatment and prevention of obesity in underserved populations. J Health Care Poor Underserved 21, 823-838.

20. Kolcic I \& Polasek O (2009) Healthy migrant effect within Croatia. Coll Antropol 33, Suppl. 1, 141-145.

21. Liu JH, Probst JC, Harun N et al. (2009) Acculturation, physical activity, and obesity among Hispanic adolescents. Ethn Health 14, 509-525.

22. Park SY, Murphy SP, Sharma S et al. (2005) Dietary intakes and health-related behaviours of Korean American women born in the USA and Korea: the multiethnic cohort study. Public Health Nutr 8, 904-911.

23. Olvera N, Suminski R \& Power TG (2005) Intergenerational perceptions of body image in Hispanics: role of BMI, gender, and acculturation. Obes Res 13, 1970-1979.

24. Nicolaou M, Doak CM, van Dam RM et al. (2009) Cultural and social influences on food consumption in Dutch residents of Turkish and Moroccan origin: a qualiltative study. J Nutr Educ Behav 41, 232-241.

25. Landman J \& Cruickshank JK (2001) A review of ethnicity, health and nutrition-related diseases in relation to migration in the United Kingdom. Public Health Nutr 4 , 647-657.

26. Mejean C, Deschamps V, Bellin-Lestienne C et al. (2010) Associations of socioeconomic factors with inadequate dietary intake in food aid users in France (the ABENA study 2004-2005). Eur J Clin Nutr 64, 374-382.

27. Labree LJ, van de Mheen $\mathrm{H}$, Rutten FF et al. (2011) Differences in overweight and obesity among children from migrant and native origin: a systematic review of the European literature. Obes Rev 12, e535-e547.

28. Kumar NB, Yu D, Akinremi TO et al. (2009) Comparing dietary and other lifestyle factors among immigrant Nigerian men living in the US and indigenous men from Nigeria: potential implications for prostate cancer risk reduction. J Immigr Minor Health 11, 391-399.

29. Harriss LR, English DR, Powles J et al. (2007) Dietary patterns and cardiovascular mortality in the Melbourne Collaborative Cohort Study. Am J Clin Nutr 86, 221-229.

30. Neuhouser ML, Thompson B, Coronado GD et al. (2004) Higher fat intake and lower fruit and vegetables intakes are associated with greater acculturation among Mexicans living in Washington State. J Am Diet Assoc 104, 51-57.

31. Renzaho AM, Swinburn B \& Burns C (2008) Maintenance of traditional cultural orientation is associated with lower rates of obesity and sedentary behaviours among African migrant children to Australia. Int J Obes (Lond) 32, 594-600. 
32. Satia-Abouta J, Patterson RE, Kristal AR et al. (2002) Psychosocial predictors of diet and acculturation in Chinese American and Chinese Canadian women. Ethn Health 7, 21-39.

33. Bundesamt für Statistik (2010) Statistik der Bevölkerung und der Haushalte (STATPOP). Neuchâtel: Swiss Federal Statistical Office.

34. Zwahlen M, Keel F, Zybach U (2006) Evaluation. Früchteund Gemüsekonsum in der Schweiz 2005 mit einem Vergleich zur „Stichtagserhebung zum täglichen Früchte- und Gemüsekonsum 2011/2002“ und zum „Food and Beverage Monitor" 2002. http://www.5amtag.ch/wp-content/uploads/ 2010/03/Bericht-Evaluation-Früchte-Gemüsekonsum-2005.pdf (accessed September 2011).

35. Allison PD (2001) Missing Data. Thousand Oaks, CA: Sage.

36. Sofianou A, Fung TT \& Tucker KL (2011) Differences in diet pattern adherence by nativity and duration of US residence in the Mexican-American population. J Am Diet Assoc $\mathbf{1 1 1}$, 1563-1569.

37. Shin CN \& Lach H (2011) Nutritional issues of Korean Americans. Clin Nurs Res 20, 162-180.

38. Gregory-Mercado KY, Staten LK, Ranger-Moore J et al. (2006) Fruit and vegetable consumption of older MexicanAmerican women is associated with their acculturation level. Ethn Dis 16, 89-95.

39. Grossmann F, Leventhal ME, Auer-Böer B et al. (2011) Selfreported cardiovascular risk factors in immigrants and Swiss nationals. Public Health Nurs 28, 129-139.

40. Figueiredo I, Jaime PC \& Monteiro CA (2008) Factors associated with fruit and vegetable intake among adults of the city of São Paulo, Southeastern Brazil. Rev Saude Publica 42, 1-8.

41. Dowler E (2001) Inequalities in diet and physical activity in Europe. Public Health Nutr 4, 701-709.

42. Agudo A (2005) Measuring Intake of Fruit and Vegetable. Background Paper for the Joint FAO/WHO Workshop on Fruit and Vegetables for Health, 1-3 September 2004, Kobe, Japan. Geneva: WHO

43. MacIntyre U, Venter C \& Vorster H (2001) A culturesensitive quantiative food frequency questionnaire used in an African population: 2. Relative validation by 7-day weighted records and biomarkers. Public Health Nutr 4, 63-71.

44. Agudo A, Slimani N, Ocke MC et al. (2002) Consumption of vegetables, fruit and other plant foods in the European Prospective Investigation into Cancer and Nutrition (EPIC) cohorts from 10 European countries. Public Health Nutr $\mathbf{5}$, 1179-1196.

45. Kim DJ \& Holowaty EJ (2003) Brief, validated survey instruments for the measurement of fruit and vegetable intakes in adults: a review. Prev Med 36, 440-447.

46. Ocke MC, Bueno-de-Mesquita H, Goddijn H et al. (1997) The Dutch EPIC food frequency questionnaire. I. Description of the questionnaire, and relative validity and reproducibility for food groups. Int J Epidemiol 26, Suppl. 1, S37-S48.

47. Pisani P, Faggiano F, Krogh V et al. (1997) Relative validity and reproducibility of a food frequency dietary questionnaire for use in the Italian EPIC centres. Int J Epidemiol 26, Suppl. 1, S152-S160.

48. Brug J (2008) Determinants of healthy eating: motivation, abilities and environmental opportunities. Fam Pract 25, Suppl. 1, i50-i55.

49. Darmon N, Briend A \& Drewnowski A (2004) Energydense diets are associated with lower diet costs: a community study of French adults. Public Health Nutr 7, 21-27.

50. Dibsdall LA, Lambert N, Bobbin RF et al. (2003) Lowincome consumers' attitudes and behaviour towards access, availability and motivation to eat fruit and vegetables. Public Health Nutr 6, 159-168.

51. Birch LL (1998) Development of food acceptance patterns in the first years of life. Proc Nutr Soc 57, 617-624.

52. Birch LL \& Fisher JO (1998) Development of eating behaviors among children and adolescents. Pediatrics 101, 539-549.

53. Birch LL, Gunder L, Grimm-Thomas K et al. (1998) Infants' consumption of a new food enhances acceptance of similar foods. Appetite 30, 283-295.

54. Wardle J, Cooke LJ, Gibson EL et al. (2003) Increasing children's acceptance of vegetables; a randomized trial of parent-led exposure. Appetite 40, 155-162.

55. Wardle J, Herrera ML, Cooke L et al. (2003) Modifying children's food preferences: the effects of exposure and reward on acceptance of an unfamiliar vegetable. Eur J Clin Nutr 57, 341-348.

56. Bandura A (1997) Self-Efficacy: The Excercise of Control. New York: Freeman.

57. Rasmussen M, Krolner R, Klepp KI et al. (2006) Determinants of fruit and vegetable consumption among children and adolescents: a review of the literature. Part I: Quantitative studies. Int J Behav Nutr Phys Act 3, 22.

58. Shaikh AR, Yaroch AL, Nebeling L et al. (2008) Psychosocial predictors of fruit and vegetable consumption in adults a review of the literature. Am J Prev Med 34, 535-543.

59. Guillaumie L, Godin G \& Vezina-Im LA (2010) Psychosocial determinants of fruit and vegetable intake in adult population: a systematic review. Int J Behav Nutr Phys Act 7, 12.

60. Swinburn B, Egger G \& Raza F (1999) Dissecting obesogenic environments: the development and application of a framework for identifying and prioritizing environmental interventions for obesity. Prev Med 29, 563-570.

61. Schuler M, Dessemontet P \& Joye D (2005) Die Raumgliederung der Schweiz. Neuchâtel: Federal Statistical Office. 